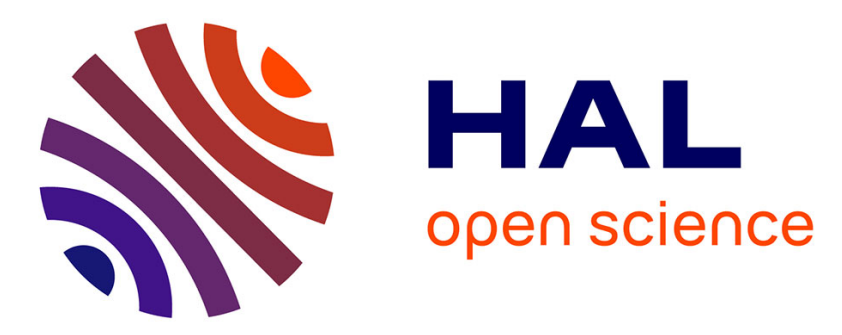

\title{
Altération d'habitat hydraulique à l'échelle des bassins versants: impacts des prélèvements en nappe du bassin Seine-Normandie
}

Corallie Miguel, Nicolas Lamouroux, Herve Pella, Baptiste Labarthe, Nicolas

Flipo, Maïa Akopian, Jérôme Belliard

\section{To cite this version:}

Corallie Miguel, Nicolas Lamouroux, Herve Pella, Baptiste Labarthe, Nicolas Flipo, et al.. Altération d'habitat hydraulique à l'échelle des bassins versants: impacts des prélèvements en nappe du bassin Seine-Normandie. La Houille Blanche - Revue internationale de l'eau, 2016, 3, pp.65-74. 10.1051/lhb/2016032 . hal-01399971

\section{HAL Id: hal-01399971 https://hal.science/hal-01399971}

Submitted on 21 Nov 2016

HAL is a multi-disciplinary open access archive for the deposit and dissemination of scientific research documents, whether they are published or not. The documents may come from teaching and research institutions in France or abroad, or from public or private research centers.
L'archive ouverte pluridisciplinaire HAL, est destinée au dépôt et à la diffusion de documents scientifiques de niveau recherche, publiés ou non, émanant des établissements d'enseignement et de recherche français ou étrangers, des laboratoires publics ou privés. 


\title{
Altération d'habitat hydraulique à l'échelle des bassins versants: impacts des prélèvements en nappe du bassin Seine-Normandie
}

\author{
Coralie MIGUEL ${ }^{1}$, Nicolas LAMOUROUX'1, Hervé PELLA', Baptiste LABARTHE², Nicolas FLIPO², \\ Maia AKOPIAN ${ }^{3}$, Jérôme BELLIARD ${ }^{4}$
}

\author{
${ }^{1}$ IRSTEA UR MALY: nicolas.lamouroux@irstea.fr ; hervé.pella@irstea.fr \\ 2. MINES ParisTech, PSL Research University, Centre de Géosciences : nicolas.flipo@mines-paristech.fr \\ 3. Agence de l'Eau Seine-Normandie : akopian.maia@aesn.fr \\ 4. IRSTEAUR HBAN : jerome.belliard@irstea.fr
}

\begin{abstract}
RÉSUMÉ. - Parmi les outils permettant d'évaluer les impacts écologiques des altérations de débit des cours d'eau, les modèles d'habitat hydraulique couplent une modélisation hydraulique avec des modèles des exigences écologiques des espèces aquatiques. La simplification de ces modèles permet de les appliquer à l'échelle des bassins versants pour quantifier l'impact sur l'habitat hydraulique de la gestion des débits et des prélèvements. Nous réalisons sur le bassin Seine-Normandie un couplage original d'un modèle hydrologique distribué, de modèles de distribution spatiale des poissons et de modèles d'habitat simplifiés. Ce couplage montre que les prélèvements en nappe génèrent une altération des débits d'étiage modérée (médiane: $4.1 \%$ ) qui se traduit pour les espèces en place par une altération d'habitat favorable faible (médiane: $1.6 \%$ ). Néanmoins, la répartition spatiale des altérations permet d'identifier les territoires les plus impactés (altération d'habitat $\sim 15 \%$ ), du fait de prélèvements importants et/ou de la présence d'espèces plus exigeantes. Une analyse des incertitudes suggère d'interpréter les résultats à l'échelle de territoires ou plus large, et l'exercice permet donc d'identifier les territoires sur lesquels une modélisation plus fine serait utile. Ces résultats ouvrent de nombreuses perspectives pour prendre en compte les milieux aquatiques dans la gestion quantitative des bassins.
\end{abstract}

Mots-clés : ecohydraulique, prélèvements souterrains, habitat des poissons

\section{Hydraulic habitat alteration at the catchment scale: impacts of groundwater abstraction in the Seine-Normandie catchment}

\begin{abstract}
Among available tools for estimating the ecological impacts of discharge alteration, hydraulic habitat models link hydraulic models of streams with biological models that reflect the preferences of organisms for hydraulics. The simplification of habitat models now enables their application at the catchment scale for estimating the impacts on habitats of discharge and abstraction management. In the Seine-Normandie catchment in France, we propose an original linkage of a distributed hydrological model, a fish species distribution model and hydraulic habitat models. We show that groundwater abstraction in the catchment cause a moderate alteration of low flows (median: 4.1\%) and a weak alteration of usable habitats for fish (median: 1.6\%). However, the spatial distribution of alterations helps to identify regions where impacts are stronger (habitat alteration around 15\%), due to strong abstraction and/or the presence of demanding fish species. An uncertainty analysis indicates that our results should be interpreted at the regional scale only; they allow identifying regions that would deserve more detailed analyses. Our results open many perspectives for taking into account habitat issues in quantitative water management at the catchment scale.
\end{abstract}

Key-words: ecohydraulics, groundwater abstraction, fish habitat

\section{INTRODUCTION}

Afin d'évaluer les impacts écologiques des altérations de débits, plus de 200 méthodes ont été développées à travers le monde, réparties en trois approches [Tharme, 2003]. La première approche est celle des méthodes « hydrologiques », qui quantifient l'altération des caractéristiques du régime hydrologique d'intérêt écologique (débits fréquents, périodes d'étiage, crues et leur intensité, durée...) par rapport à une situation naturalisée [Richter et al., 1996 ; Poff et al., 2010]. Les méthodes dites " hydrauliques » consistent à étudier comment les altérations de débit se traduisent en altérations de variables hydrauliques (vitesses du courant, hauteur d'eau, contraintes sur le lit, périmètre mouillé). Leur avantage est de prendre en compte la morphologie du cours d'eau étudié, et de décrire les variables d'habitat des espèces de façon plus pertinente que le seul débit. Enfin, les méthodes dites « habitats » couplent la modélisation hydraulique du cours d'eau avec des modèles biologiques, dits de "préférences », qui prennent en compte les exigences écologiques des espèces aquatiques suivant leur stade de vie ou leur activité [Ginot, 1998]. Il est conseillé de combiner ces approches lors des études d'impact écologique de l'altération des débits (ex : Circulaire du 5 juillet 2011 relative à l'application de l'article L. 214-18 du code de l'environnement sur les débits réservés à maintenir en cours d'eau ; NOR : DEVL1117584C).

Les modèles d'habitat sont attractifs pour quantifier les risques d'altération écologique, mais leur utilisation restait jusqu'à récemment restreinte à l'échelle des tronçons de 
cours d'eau (longueur de l'ordre $\mathrm{du} \mathrm{km}$ ) pour des raisons de coût et de complexité d'application. Cependant, de nouvelles réglementations nationales et internationales appuient la nécessité d'estimer et de limiter les altérations biologiques induites par les altérations hydrologiques à l'échelle d'un ou plusieurs bassins versants [Acreman et al., 2014 ; Wilding et al., 2014]. En particulier, la Directive Cadre européenne sur l'Eau (DCE) de 2000 impose l'intégration de la qualité des milieux aquatiques de surface dans l'évaluation de l'état des masses d'eau souterraines. Elle demande d'estimer les quantités et les directions des flux entre les masses d'eau souterraines et superficielles, et de déterminer si les pressions de prélèvements en nappe ont des impacts significatifs sur les écosystèmes aquatiques. Par ailleurs, la circulaire française du 30 juin 2008 (relative à la résorption des déficits quantitatifs en matière de prélèvements d'eau et de gestion collective des prélèvements d'irrigation) préconise de définir des volumes prélevables par saison tout en préservant la vie aquatique. Cette circulaire met en évidence la nécessité d'estimer les volumes que le milieu est réellement capable de fournir tout en respectant des conditions écologiques satisfaisantes.

L'utilisation des modèles d'habitat à l'échelle des bassins devient aujourd'hui possible pour deux raisons principales [Lamouroux, 2008a ; Snelder et al., 2011a ; Wilding et al., 2014]. En premier lieu, des modèles d'habitat simplifiés ont été développés et permettent des applications moins coûteuses, notamment en France via la méthode "Estimhab » [Lamouroux, 2008b]. Ces modèles reposent sur une modélisation statistique des sorties des modèles d'habitat classiques (courbes reliant une valeur d'habitat ou une surface utile au débit) en fonction des caractéristiques hydrauliques moyennes des cours d'eau [Lamouroux et Capra, 2002]. En second lieu, le degré de transférabilité entre cours d'eau des préférences biologiques est aujourd'hui mieux cerné et justifie, pour certaines applications, d'utiliser un même modèle de préférence à l'échelle de l'ensemble d'un bassin [Lamouroux et al., 2013]. Ainsi, l'utilisation de modèles d'habitat à l'échelle des bassins a été proposée récemment pour quantifier les impacts de différents scénarios législatif concernant les débits d'étiage en Nouvelle-Zélande [Snelder et al., 2011a]. Cette première application s'est basée sur des altérations de débits hypothétiques, faute de disposer d'estimations d'altérations hydrologiques assez précises à large échelle.
Dans cet article, nous proposons une première traduction d'altérations hydrologiques réelles en altérations d'habitat à l'échelle d'un grand bassin (Seine-Normandie) sur lequel une modélisation des altérations hydrologiques est disponible. Pour cela, nous réalisons un couplage de modèles hydrologiques distribués et de modèles d'habitat simplifiés pour les poissons. Les altérations hydrologiques concernées sont uniquement les altérations de débit d'étiage liées aux prélèvements en nappe. Nous expliquons la méthodologie générale avant de la détailler, puis nous présentons et discutons les résultats des simulations.

\section{METHODES}

\section{II.1. Principe des modèles d'habitat hydraulique et méthodologie générale}

Le principe de base des modèles d'habitat hydraulique (Figure 1) est de coupler une description hydraulique des tronçons de cours d'eau avec des modèles de préférences hydrauliques des espèces aquatiques [Ginot, 1998 ; Lamouroux, 2008b]. Le modèle hydraulique décrit les vitesses et hauteurs d'eau en fonction du débit au sein du tronçon; le modèle biologique donne pour chaque stade de vie, espèce ou groupe d'espèces, une valeur d'habitat (note entre 0 et 1) qui dépend des caractéristiques hydrauliques au sein de chaque maille du modèle hydraulique. La valeur d'habitat moyenne sur le tronçon est multipliée par la surface du tronçon (ici pour une longueur de $100 \mathrm{~m}$ ) pour obtenir une surface pondérée utile (SPU).

Notre démarche globale (Figure 2) correspond à l'application à l'échelle du bassin de modèles d'habitat simplifiés, sur support SIG (approche « Estimkart »; Lamouroux et al., 2010). Le support des calculs est un réseau hydrographique où chaque tronçon de cours d'eau est décrit à l'aide de caractéristiques environnementales (ex : pente, surface de bassin versant amont, températures mensuelles moyennes, altitude). Un modèle hydrologique distribué [Flipo et al., 2013 ; Pryet et al., sous presse] permet de calculer l'altération hydrologique, différence entre une situation «naturalisée » (sans pompages en nappe) et une situation « influencée » (avec pompages). Un modèle de géométrie hydraulique permet ensuite de traduire l'information hydrologique en information hydraulique (largeur
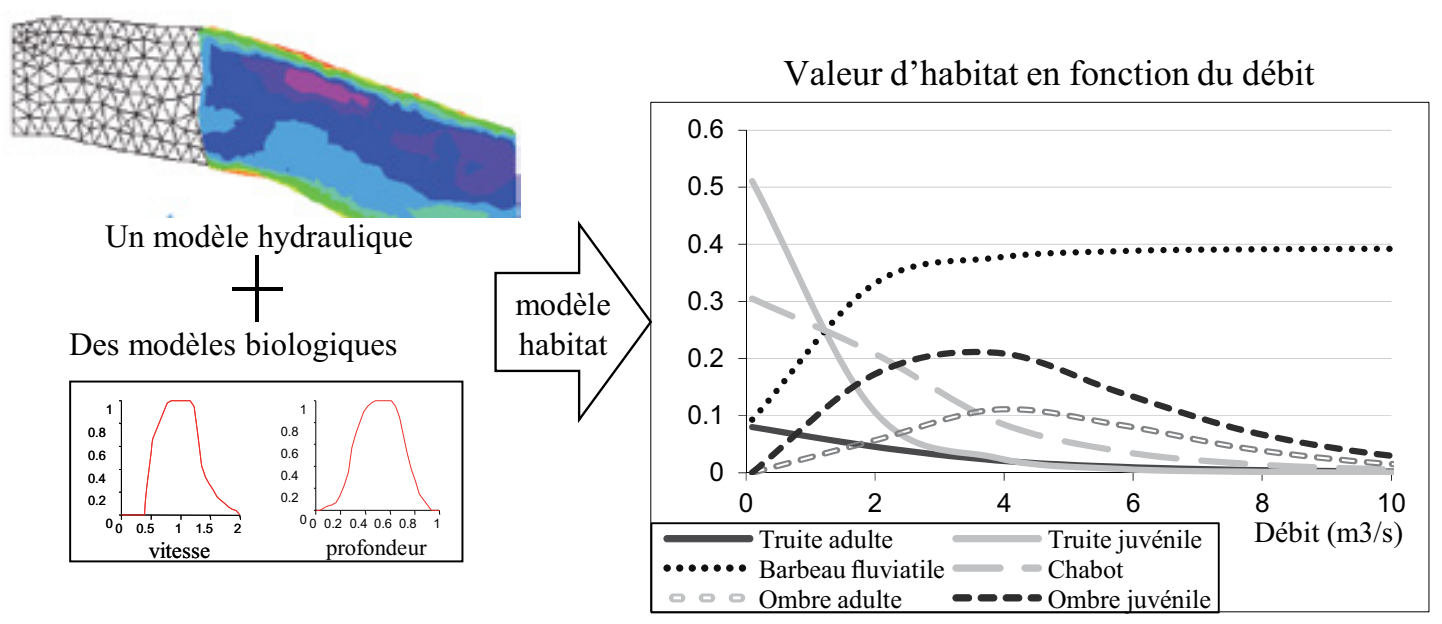

Figure 1 : Principe de base d'un modèle habitat. 


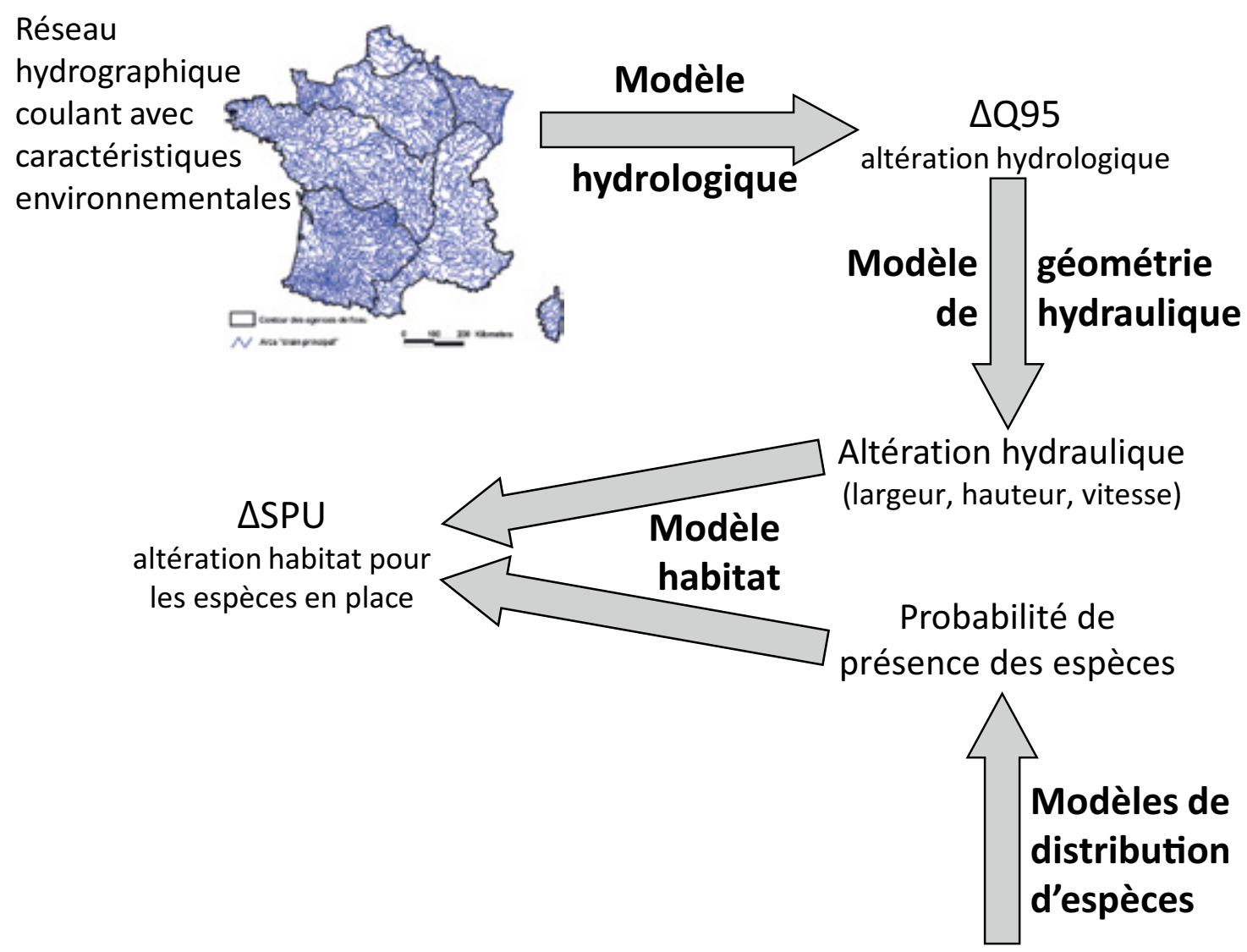

Figure 2 : Méthodologie générale.

mouillée du tronçon de cours d'eau, hauteur d'eau, vitesse). Nous utilisons alors un ensemble de modèles de distribution d'espèces afin d'établir la probabilité de présence de différentes espèces de poissons selon les caractéristiques environnementales du tronçon. Enfin, nous calculons l'altération de l'habitat hydraulique des espèces à forte probabilité de présence à l'aide de modèles d'habitat simplifiés.

Les altérations hydrologiques $(\triangle Q 95)$ sont calculées pour le quantile de débit journalier Q95 (dépassé $95 \%$ du temps) et les altérations d'habitat sont exprimées en modification de « surface utile» $(\triangle S P U)$ pour les poissons.

\section{II.2. Réseaux hydrographiques du bassin Seine Normandie considérés pour les simulations}

Trois réseaux hydrographiques ont été utilisés pour appliquer les modèles, tous dérivés du réseau BD Carthage $\AA$ de l'IGN (Institut National de l'Information Géographique et Forestière, Saint-Mandé, France, http://professionnels.ign. $\mathrm{fr} /$ bdcarthage). Le réseau le plus détaillé, utilisé pour réaliser les calculs, est le Réseau Hydrographique Théorique [RHT ; Pella et al., 2012]. Le RHT est un réseau théorique construit à partir du modèle numérique de terrain (MNT) à $50 \mathrm{~m}$ de l'IGN, reconditionné à partir de la BD Carthage ${ }^{\circledR}$. Cette procédure force le réseau théorique à être proche de la BD Carthage ${ }^{\circledR}$ mais reste compatible avec le MNT, ce qui améliore les estimations de surface de bassins drainés. Le RHT s'étend sur toute la France métropolitaine et présente un linéaire cumulé de $283639 \mathrm{~km}$ (114 601 tronçons), soit $80 \%$ du linéaire de la BD Carthage ${ }^{\circledR}$. C'est un réseau vectoriel jointif orienté de l'amont vers l'aval, ce qui rend possible le parcours et l'analyse du réseau. Un ensemble de caractéristiques morphologiques est renseigné sur le RHT [Pella et al., 2012] dont la pente moyenne du tronçon, la surface du bassin versant amont, ainsi qu'un ensemble de variables climatiques et hydrologiques. Les caractéristiques hydrologiques sont issues d'extrapolations géostatistiques des lames d'eau annuelles reconstituées reflétant une situation naturalisée, c'est à dire sans influence anthropique [Sauquet 2006 ; Sauquet et Catalogne, 2011]. Elles incluent le module (débit moyen interannuel), ainsi que le quantile de débit Q95 représentant le débit journalier statistiquement dépassé $95 \%$ du temps que nous avons utilisé dans cette étude. Une estimation de la taille moyenne des éléments du substrat est également extrapolée sur le RHT [Snelder et al., 2011b].

Le deuxième réseau considéré dans cet article (RHT_DCE) est une extraction du RHT correspondant au réseau Masse d'Eau Cours d'Eau (MECE) du bassin Seine-Normandie. La mise en cohérence de ces deux réseaux est nécessaire, car c'est à partir du réseau MECE que sont décidées et appliquées les préconisations des Schémas Directeurs d'Aménagement de Gestion des Eaux (SDAGE). Le réseau MECE est celui considéré par la Directive Cadre sur l'Eau, qui définit comme masse d'eau les tronçons homogènes des cours d'eau drainant un bassin versant d'au moins $10 \mathrm{~km}^{2}$. Ce réseau est ainsi composé de 1681 masses d'eau, naturelles ou anthropisées. Le RHT ne représentant pas les cours d'eau non naturels mais pouvant contenir des talwegs secs ou des cours d'eau au bassin versant drainé plus petit que la limite DCE, une correspondance géographique des deux réseaux a permis de créer le réseau RHT_MECE de 8988 arcs RHT équivalent à 1630 masses d'eau. Ainsi, le réseau RHT_DCE contient 
8988 tronçons du RHT correspondant à 1630 masses d'eau. Un troisième réseau (RHT_MECENa) correspond cette fois à une sélection du réseau RHT_DCE sur lequel les simulations hydrologiques ont été réalisées. Il comprend 727 masses d'eau correspondant à 5216 tronçons du RHT.

Enfin, notre étude portant sur l'impact des prélèvements en nappe, les résultats seront en général agrégés par masses d'eau souterraine (MESO) définies dans le cadre de la DCE. Chaque tronçon de réseau se voit ainsi attribuer l'identifiant de la MESO de niveau 1 (identifiée parmi 60 MESO affleurantes) sur laquelle s'écoule la plus grande partie de son linéaire.

\section{II.3. Modélisation des altérations hydrologiques}

Les altérations hydrologiques sont estimées par la plate-forme EauDyssée [Flipo et al., 2012 ; Pryet et al., soumis ; Saleh et al., 2011] qui regroupe des couplages de modèles hydrogéologiques spatialisés (maille de $1 \mathrm{~km}$ à $250 \mathrm{~m}$ ) multicouches. Les modèles estiment les échanges de flux entre les eaux souterraines et les eaux superficielles selon deux scénarios : avec ou sans prélèvements en nappe (période 1993-2010, [Flipo et al., 2013 ; Pryet et al., sous presse]). Grâce à ces simulations, les quantiles de débits journaliers du cours d'eau sont disponibles pour les scénarios avec ou sans prélèvements en nappe.

Les prélèvements en nappe affectant essentiellement la sévérité des étiages, nous nous focaliserons sur le quantile de débit journalier Q95. Ce débit d'étiage est considéré comme suffisamment fréquent (le débit est par définition inférieur à Q95 en moyenne 18 jours par an) pour structurer les peuplements aquatiques et est utilisé pour représenter les étiages dans le cadre de la DCE [Acreman et Ferguson, 2010]. L'altération hydrologique sera décrite par la modification relative de Q95 ( $\triangle Q 95$, exprimée en pourcentage)

$$
\Delta Q 95=\frac{Q 95 s p-Q 95 a p}{Q 95 s p},
$$

où $\Delta Q 95$ indique l'altération hydrologique de Q95, Q95sp indique le Q95 du scénario sans prélèvements en nappe et Q95ap indique le Q95 du scénario avec prélèvements.

Hors du réseau RHT_MECENa (sur les 903 masses d'eau non modélisées du réseau RHT_DCE et sur l'ensemble du réseau RHT), les altérations n'ont pas été calculées par le modèle hydrologique et nous les avons donc extrapolées spatialement. Pour les tronçons se trouvant au droit d'une MESO comportant déjà des tronçons RHT_MECENa, l'altération hors RHT_MECENa est fixée à la médiane des altérations hydrologiques des tronçons RHT_MECENa de la MESO. Pour les tronçons se trouvant au droit d'une MESO ne comportant aucun tronçon RHT_MECENa (4631 tronçons), l'altération hydrologique est fixée à la médiane des altérations hydrologiques des tronçons RHT_MECENa du bassin.

Le modèle hydrologique étant focalisé sur l'altération des débits liée aux prélèvements en nappe ( $\triangle Q 95$, soit la proportion de débit prélevé), nous avons par la suite considéré que les estimations de Q95 naturalisées disponibles sur le RHT étaient les plus fiables [Sauquet et Catalogne, 2011]. En conséquence, nous avons reporté les altérations estimées par le modèle hydrologique sur les estimations hydrologiques de débit naturalisé $(Q 95 n)$ calculées sur le RHT [Sauquet et Catalogne, 2011]. Ainsi, le Q95 influencé par les prélèvements souterrains $(Q 95 i)$ est :

$$
Q 95 i=Q 95 n *(1-\Delta Q 95)
$$

\section{II.4. Modélisation des altérations d'habitat hydraulique}

\section{II.4.1. Modèle de géométrie hydraulique}

Un modèle de géométrique hydraulique est utilisé afin de transformer l'information hydrologique en information hydraulique. Ce modèle va permettre une estimation, pour chaque tronçon du réseau, des valeurs de hauteur d'eau $(H)$, de largeur mouillée $(L)$ et de vitesse $(V)$ en fonction du débit $Q$. Il repose sur des lois puissance classiques [Leopold et Maddock, 1953 ; Park, 1977 ; Lamouroux, 2008a : Equations (3) et (4) en Annexe 1] qui relient d'une part $H$, $L$ et $V$ à $Q$ sur chaque tronçon (géométrie hydraulique « stationnelle », relative aux variations temporelles sur le tronçon), et d'autre part les variations de $H, L$ et $V$ au module entre tronçons (géométrie "longitudinale », relative aux variations spatiales).

\section{II.4.2. Modèles de distribution des espèces}

Afin de déterminer quelles sont les espèces théoriquement présentes dans les tronçons du bassin, nous avons utilisé les modèles prédictifs de distribution des espèces développés pour la mise au point de l'« Indice Poissons Rivière » français [Oberdorff et al., 2001]. Ces modèles estiment la probabilité de présence de différentes espèces de poissons dans un tronçon en fonction des variables environnementales disponibles sur le RHT (températures moyennes de janvier et de juillet, distance à la source, pente du tronçon, altitude et surface de bassin) ou calculées ci-dessus ( $H$ et $L$ au module). Cinq espèces ou groupes biologiques, sensibles aux diminutions de débit, ont été retenus ici (Tableau 1). Des seuils de probabilité par groupe ont été mis en place pour considérer une espèce ou un groupe d'espèces comme potentiellement présent (Tableau 1). Ces seuils sont inspirés

Tableau 1 : Groupes biologiques pour lesquels seront réalisées les simulations d'habitat, et seuils de probabilité de présence retenus pour considérer le groupe.

\begin{tabular}{|c|c|c|}
\hline Nom du groupe & Espèces (stades modélisés) & Seuil \\
\hline TRF & Truite (TRFa : adulte, TRFj : juvenile \& alevin) & 0.8 \\
\hline CHA & Chabot (adultes) & 0.4 \\
\hline OBR & Ombre commun (OBRa : adulte, OBRj : juvenile, OBRal: alevin) & 0.2 \\
\hline Espèces d'Eau Courante (EEC) & Ablette, Barbeau fluviatile, Hotu, Vandoise (adultes) & 0.2 \\
\hline BAF & Barbeau fluviatile (adultes) & 0.4 \\
\hline
\end{tabular}


des seuils proposés par Logez et al., [2012], dont le principe est de maximiser la somme de la sensibilité (probabilité que le modèle détecte la présence de l'espèce) et de la spécificité (probabilité de détecter l'absence) des modèles probabilistes [Fiedling et Bell, 1997], car le choix d'un seuil de probabilité " équilibré » entre présence et absence dépend entre autres de la prévalence (vraie occurrence moyenne) du groupe. Pour le groupe des espèces d'eau courante, le seuil de 0.2 est un compromis entre les valeurs des différentes espèces du groupe. Le barbeau fluviatile fait partie de ce groupe. Néanmoins, nous avons réalisé une modélisation spécifique pour le barbeau lorsque sa probabilité de présence était forte (seuil fixé à 0.4).

\section{II.4.3. Modèles d'habitat simplifiés}

Nous avons utilisé le modèle d'habitat hydraulique statistique Estimhab [Lamouroux et Capra, 2002 ; Lamouroux, 2008b]. Estimhab est issu d'une analyse de sensibilité de modèles d'habitat numériques (Evha ; [Ginot, 1998]) et a pour variables d'entrée les caractéristiques de géométrie hydraulique décrites ci-dessus et deux autres attributs du RHT : le débit médian inter-annuel et la taille moyenne des éléments du substrat.

Pour chaque groupe d'espèces (et pour chaque stade de développement en ce qui concerne la truite et l'ombre), nous calculons une SPU au débit $Q 95 n$ naturalisé (SPUn) et au débit $Q 95 i$ influencé (SPUi). L'altération d'habitat s'en déduit (exprimée en pourcentage) :

$$
\triangle S P U=\frac{S P U n-S P U i}{S P U n},
$$

L'altération d'habitat n'a de sens que pour les espèces potentiellement présentes, et c'est ici qu'intervient le modèle théorique de peuplement. Sur chaque tronçon, nous retenons l'altération d'habitat maximum pour les groupes biologiques théoriquement présents sur le tronçon. Le groupe " limitant » (pour lequel l'altération d'habitat est maximale) est identifié.

\section{II.4.4. Incertitudes}

Le modèle hydrologique comme la chaine de modèles « Estimkart » sont extrapolés à l'ensemble du réseau hydrographique ce qui implique naturellement la propagation des incertitudes des données d'entrée et des modèles. Ces incertitudes peuvent être très fortes à l'échelle d'un tronçon et doivent être quantifiées pour cadrer l'interprétation. Par exemple, un intervalle de confiance à $95 \%$ concernant un module estimé à $0.2 \mathrm{~m}^{3} / \mathrm{s}$ sur un tronçon du RHT est de l'ordre de $\pm 100 \%$ [Lamouroux et al., 2014]. Ici, les incertitudes sur les simulations d'habitat sont calculées à partir d'une estimation de type Monte-Carlo. Ainsi, nous propageons sur chaque tronçon des erreurs aléatoires concernant (1) l'estimation du Q95 naturalisé $(Q 95 n)$, (2) l'estimation des altérations hydrologiques de Q95 et (3) l'estimation des grandeurs hydrauliques (coefficients et exposants de l'Annexe 1). Nous nous sommes basés ici sur les équations de Lamouroux et al. [2014] concernant le débit pour estimer les incertitudes de Q95 et sur les incertitudes issues des tests de terrain [Lamouroux et al., 2010] pour la géométrie hydraulique. Les modèles d'incertitude sont contextualisés, c'est à dire qu'ils prennent en compte comment les incertitudes varient avec les caractéristiques du tronçon. Le modèle appliqué à Q95 est originellement développé pour le module, mais la valeur du débit lui-même étant l'explication principale de l'incertitude, il est raisonnable de l'utiliser pour d'autres quantiles de débits. En l'absence d'estimation détaillée concernant les incertitudes des altérations de Q95, une hypothèse d'erreur d'un facteur 2 (erreur factorielle) est faite sur chaque tronçon.

\section{RÉSULTATS}

\section{III.1. Résultats}

Les résultats chiffrés, ici cartographiés, sont disponibles sur demande aux auteurs. Les altérations hydrologiques du Q95 (Figure 3a) mettent en lumière l'impact relativement limité des prélèvements en nappe sur les débits de cours d'eau en étiage, avec une grande majorité d'arcs pour lesquels l'altération de Q95 est inférieure à $10 \%$ (médiane $4.1 \%$ sur le réseau RHT_MECENa).

Les altérations d'habitat (Figure 3b) sont généralement plus faibles, le plus souvent inférieures à $5 \%$ (médiane $1.6 \%$ ). La comparaison des deux cartes (Figures 3a, 3b) permet d'observer une nette atténuation générale des pourcentages d'une carte à l'autre, soulignant ainsi un lissage des altérations hydrologiques par l'altération habitat.

Il existe néanmoins des zones localisées où des altérations hydrologiques modérées (entre 15 et $30 \%$ ) à fortes (supérieures à $30 \%$ ) sont recensées (par exemple dans la région de Sens, ou entre Chartres et Rouen). Certaines MESO sont particulièrement touchées comme la 3209 «Craie du Sénonais et Pays d'Othe », ou la MESO 4135 « Calcaires tertiaires captifs de Beauce sous forêt d'Orléans " au droit de laquelle la médiane de l'altération hydrologique est la plus forte $(19.7 \%)$.

De même, les altérations d'habitat sont plus importantes dans certaines zones : par exemple autour de Sens (altération entre 10 et $20 \%$ ) ou encore au sud de la MESO 3103 « Tertiaire - Champigny - en Brie et Soissonnais ». La MESO avec la médiane d'altération d'habitat maximale la plus haute $(8.6 \%)$ est la MESO 3216 « Albien-néocomien libre entre Yonne et Seine ».

Le bassin semble donc subir, en moyenne, un impact relativement limité de la pression de prélèvements souterrains, mais les simulations suggèrent des impacts plus forts à l'échelle infra-MESO.

La carte des espèces « limitantes » (dont l'altération d'habitat est maximale parmi les espèces présentes : Figure 4) indique que le barbeau fluviatile est, parmi les espèces considérées, la plus fréquemment limitante sur les grands axes hydrologiques (Seine, Marne, Aisne, Oise, Eure). Sur les axes secondaires, le groupe des espèces d'eau courante et le chabot sont les plus «limitants». Sur les quelques tronçons (63 sur le réseau RHT_DCE) où l'ombre est jugé potentiellement présent, l'ombre devient systématiquement l'espèce limitante. Cela reflète une très forte sensibilité de l'espèce aux altérations de débit. Mais il s'agit ici d'avantage d'un impact potentiel dans la mesure où l'ombre commun, introduit sur le bassin, y présente une distribution spatiale très morcelée qui ne coïncide pas toujours avec les tronçons identifiés ici.

L'espèce retenue peut influencer fortement la traduction de l'altération hydrologique en habitat. Les groupes d'espèce ont des préférences d'habitat hydraulique différentes et donc des altérations d'habitat différentes en réponse à une altération hydrologique identique. Par exemple, la MESO 3209 (Figure 5) présente une altération hydrologique médiane 


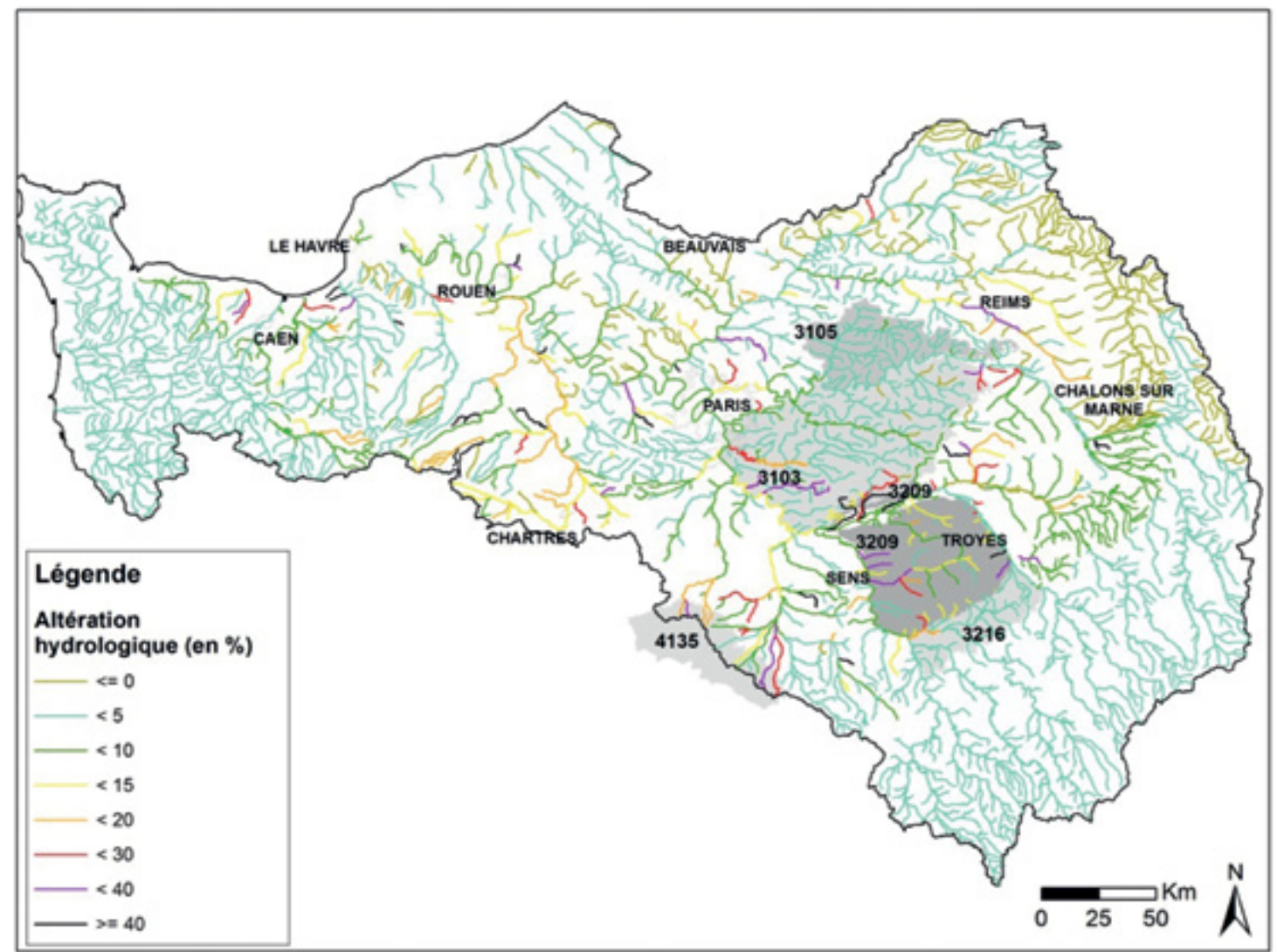

Figure 3a : Altération hydrologique sur le réseau RHT_DCE. Carte représentative de l'altération hydrologique ( $\triangle Q 95$, en \%) calculée sur le réseau RHT_DCE. Certaines MESO discutées dans l'article sont identifiées par un code.

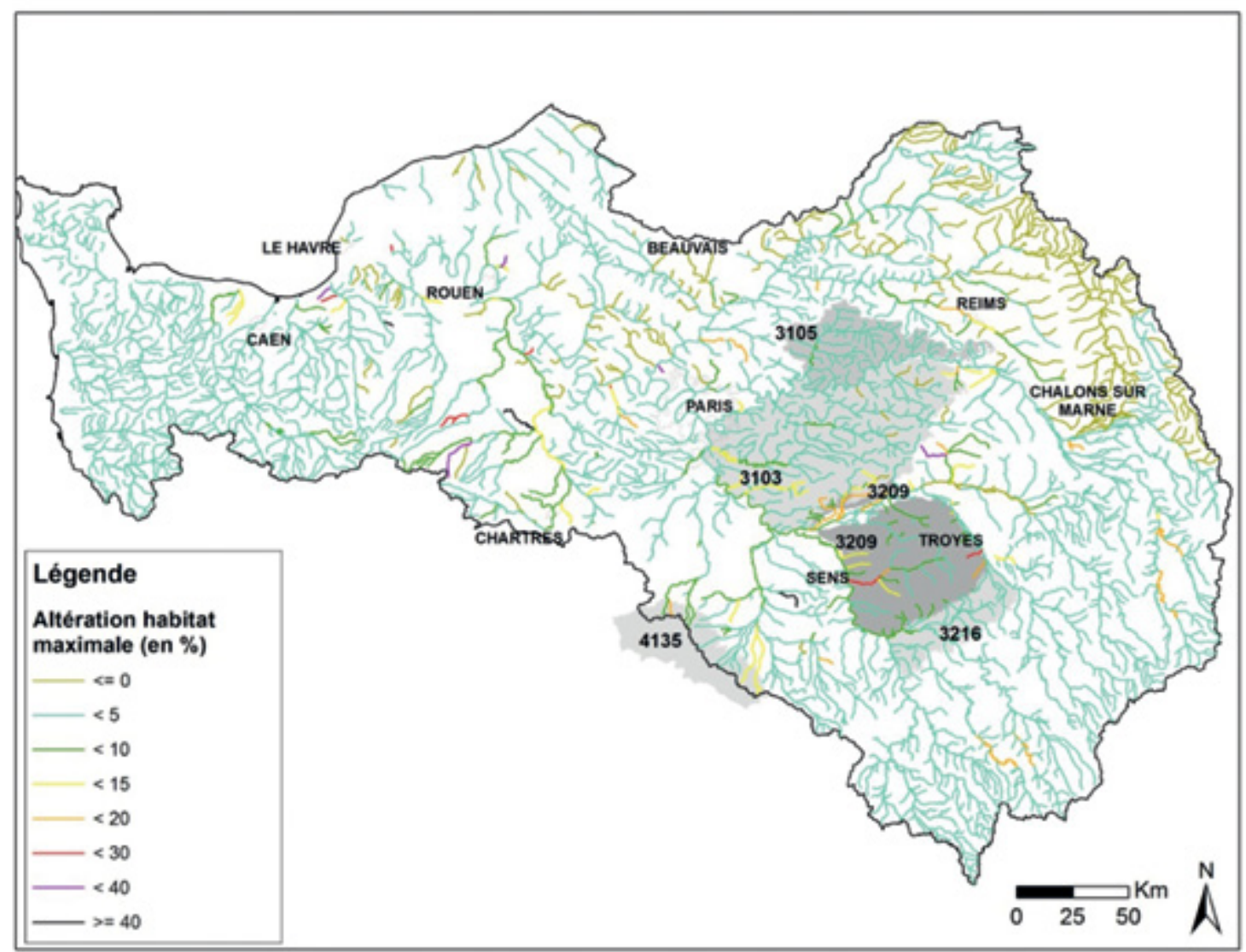

Figure 3b : Altération habitat maximale sur le réseau RHT_DCE. Carte représentative de l'altération habitat maximale $(\triangle S P U$, en \%) calculée sur le réseau RHT_DCE. 


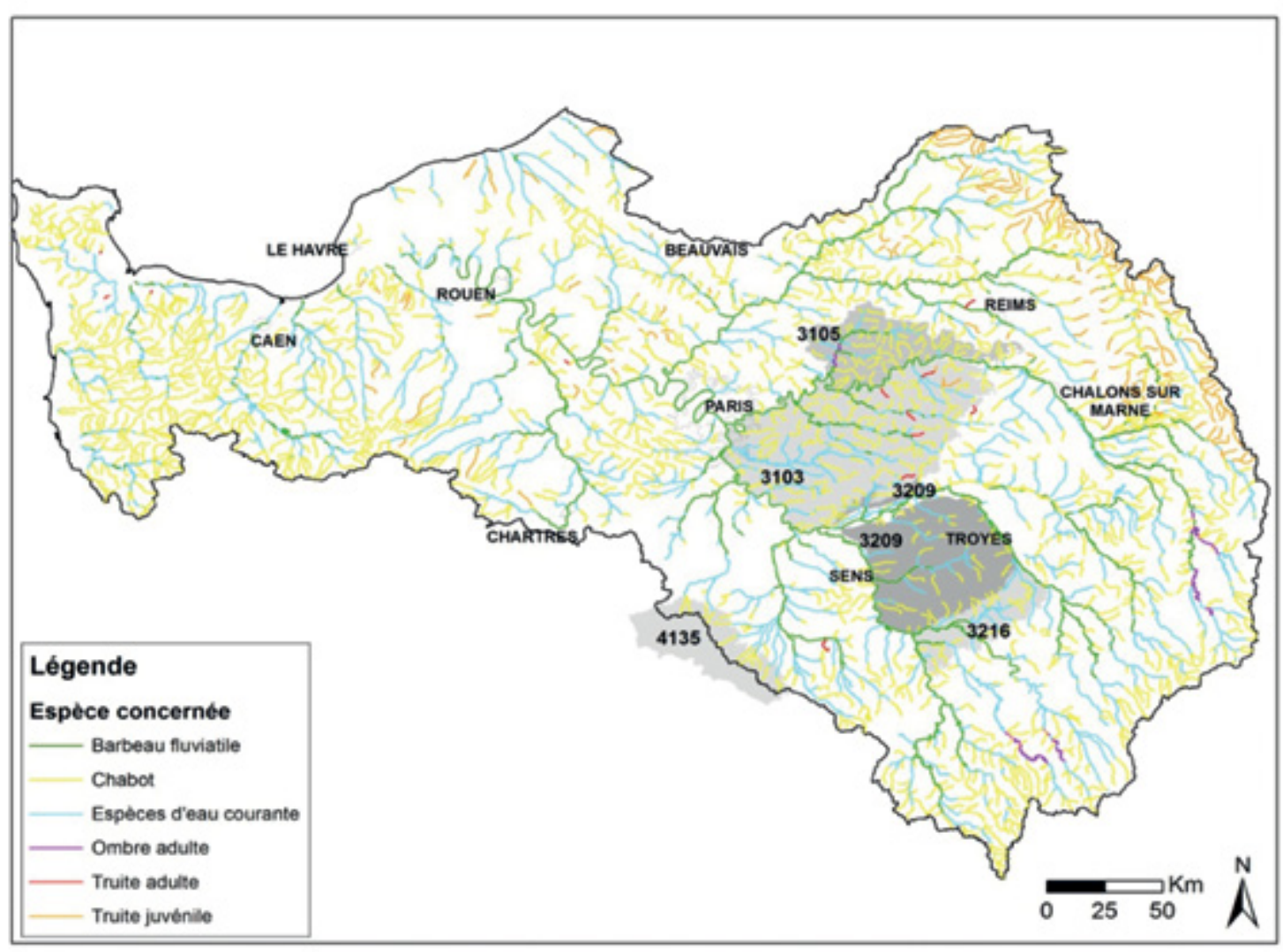

Figure 4 : Groupe biologique correspondant à l'altération d'habitat maximale.
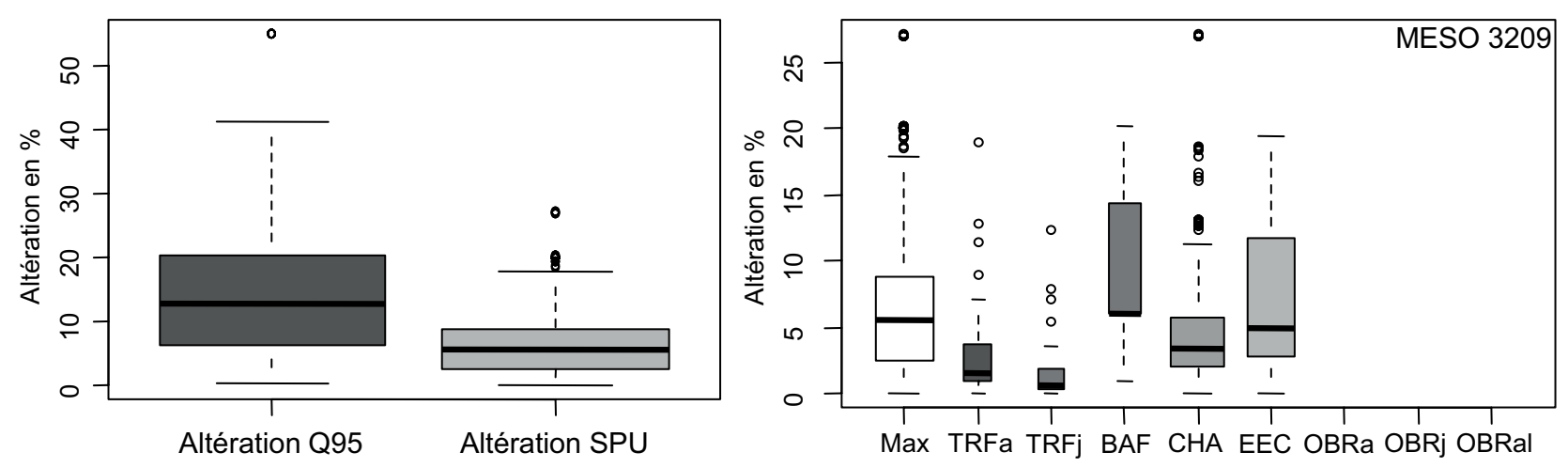

Figure 5 : Résultats d'altérations à l'échelle de la MESO 3209 : Craie su Sénonais et Pays d'Othe. Le graphique de gauche représente la distribution de l'altération hydrologique $(\triangle Q 95$, en \%) et de l'altération d'habitat maximale par tronçon ( $\triangle S P U)$. Le graphique de droite permet de comparer la distribution d'altération maximale par tronçon avec les distributions d'altération de chaque espèce ou stade (signification des codes espèce : tableau 1), avant sélection de l'altération maximale. Les boîtes à moustaches représentent la médiane (trait épais) et s'étendent du quartile 0.25 au quartile 0.75 ; les moustaches s'étendent jusqu'à la valeur distante d'au maximum 1.5 fois la distance interquartile. Les valeurs situées au-delà de cette distance sont représentées par des points.

de $12.9 \%$ (avec un minimum de $0.2 \%$ et un maximum de $55.1 \%$ ) et une altération d'habitat maximale médiane de $5.5 \%$ (minimum : 0.1, maximum : $27.1 \%$ ). Les altérations d'habitat calculées pour l'ensemble des groupes sur cette MESO (avant sélection de l'altération maximale) indiquent une médiane d'altération habitat d'environ $0.5 \%$ pour les truites juvéniles tandis que celle des barbeaux fluviatiles est d'environ $6 \%$. Ceci se confirme avec le cas de l'ombre commun. En effet, l'exemple de la MESO 3105 (Figure 6) montre que malgré une altération hydrologique maximale inférieure à $6 \%$ (Annexe 2$)$, les altérations d'habitat varient entre $5.5 \%$ et $8.9 \%$ pour les trois stades de l'ombre. 

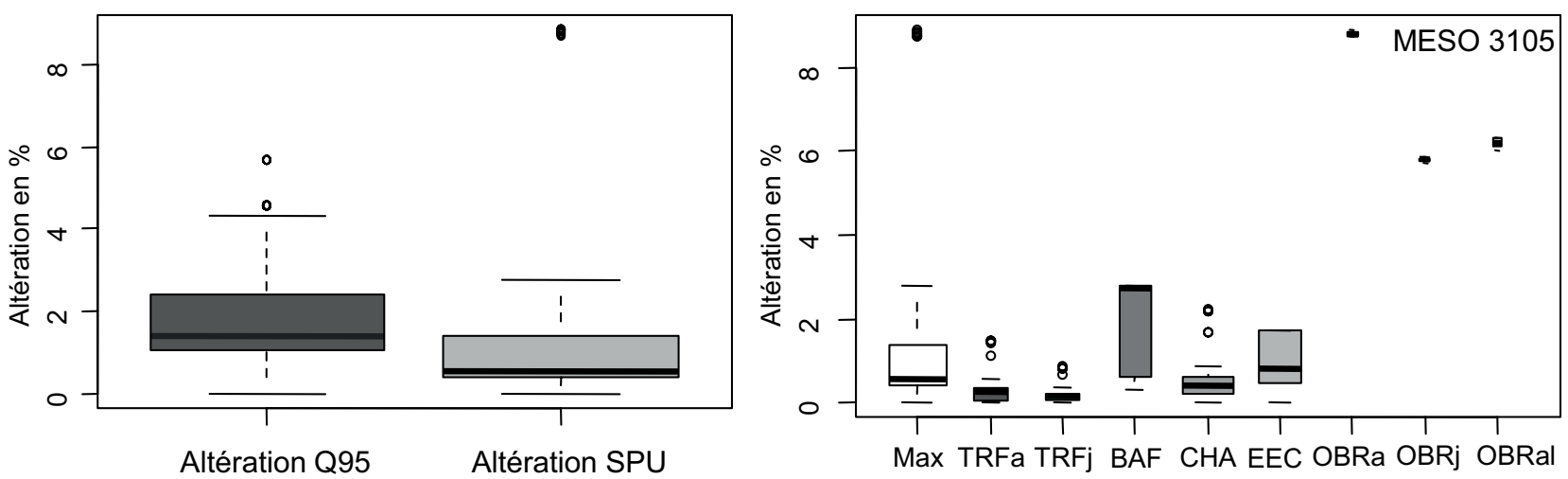

Figure 6 : Résultats d'altérations à l'échelle de la MESO 3105 : Eocène du bassin versant de l'Ourcq. Même représentation que sur la Figure 5.

\section{III.2. Incertitudes}

Les simulations d'incertitudes indiquent que les incertitudes concernant l'altération d'habitat hydrologique sont extrêmement fortes à l'échelle du tronçon (Figure 7a). En revanche, à une échelle agrégée comme celle de la MESO, les distributions d'altération d'habitat sont interprétables et comparables. Les différences entre deux MESOs (MESO 3103 avec une altération habitat médiane de $0.5 \%$ et MESO 3209 avec une altération habitat médiane de $5.5 \%$ ) restent évidentes lorsque des incertitudes sont propagées (Figure $7 \mathrm{~b}$ et $7 \mathrm{c}$ ), quel que soit le réseau hydrographique considéré.

\section{DISCUSSION ET CONCLUSIONS}

Le couplage d'une modélisation hydrologique distribuée (EauDyssée) avec des modèles d'habitat hydraulique sur SIG (Estimkart) permet une estimation globale des altérations d'habitat hydraulique des poissons à l'échelle du bassin Seine Normandie. Il faut interpréter ces simulations à une échelle des MESOs ou de sous-bassins, du fait des incertitudes fortes de l'exercice de modélisation à l'échelle d'un tronçon donné.

Sur le bassin Seine-Normandie, les simulations permettent d'apprécier que les altérations d'habitat (médiane de $1.6 \%$ ) liées aux prélèvements en nappe (médiane d'altération du Q95 de $4.1 \%$ ) sont globalement limitées. La plus forte altération d'habitat médiane d'une MESO affleurante est de $8.6 \%$. Néanmoins certaines zones (cf. Figure $3 \mathrm{~b}$ ) comme la région de Sens ou le sud de la MESO «Tertiaire - Champigny - en Brie et Soissonnais » peuvent présenter des altérations d'habitat supérieures à $15 \%$, et mériteraient des études plus poussées avec calage de terrain plus précis. Bien que les connaissances soient limitées pour estimer un seuil d'altération d'habitat qui mette en danger les objectifs de la DCE, une altération d'habitat de cet ordre est parfois considérée comme significative. Notons d'ailleurs que d'autres experts ont considéré une altération du Q95 de l'ordre de $15 \%$ comme limitante pour les objectifs de la DCE [Acreman et Ferguson, 2010].

Les exercices de modélisation à l'échelle d'un bassin souffrent inévitablement de limites multiples qu'il faut rappeler. Bien que nous ayons essayé de les quantifier ici,
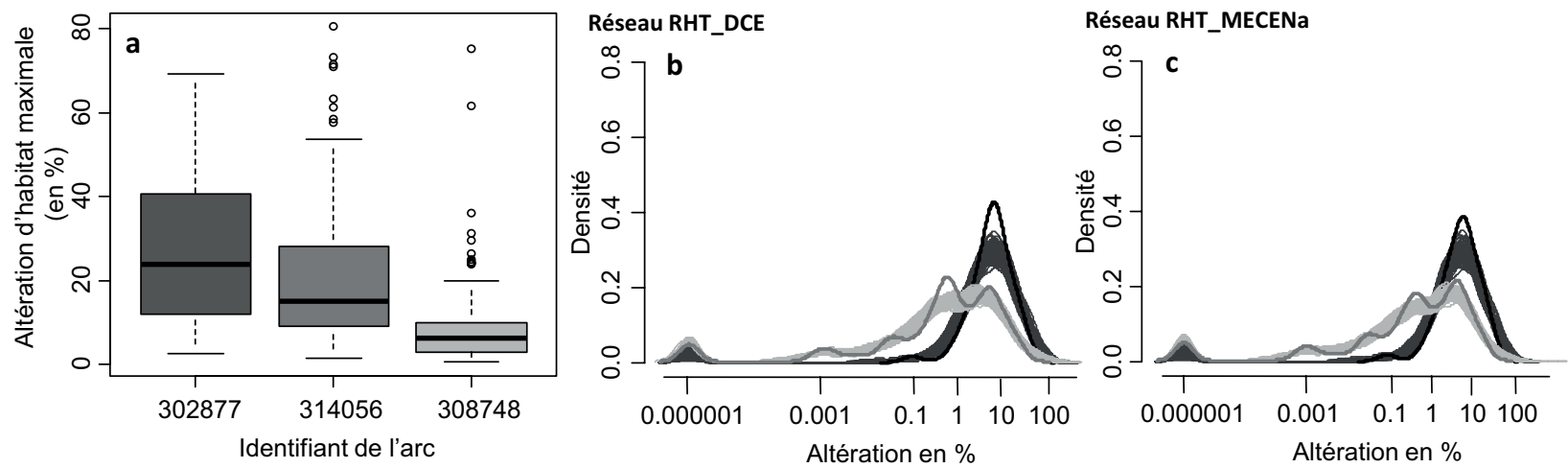

Figure 7 : Incertitudes des altérations calculées (a) à l'échelle d'un tronçon, et (b,c) à l'échelle d'une MESO pour deux réseaux hydrographiques considérés. Les altérations d'habitat estimées sur 3 tronçons (a) se différencient difficilement après propagation des incertitudes (les boites à moustaches correspondent à $n=100$ valeurs d'altération). Au contraire, les distributions des altérations d'habitat (échelle log) sur une MESO (MESO 3209 en gris foncé ; MESO 3103 en gris clair) restent stables et différenciables après propagation des incertitudes, que ce soit sur le réseau RHT_DCE (b) ou le réseau RHT_MECENa (c). Les courbes sont les distributions d'altération sur la MESO et les faisceaux correspondent à des simulations identiques après propagation des incertitudes $(n=100)$. 
les incertitudes sont fortes et parfois mal connues, comme l'incertitude de l'anomalie hydrologique dont la précision souffre de la difficulté de reconstruire des débits naturalisés. Les modèles d'habitat sont des visions simplificatrices $\mathrm{du}$ cycle de vie complexe des organismes aquatiques, ne concernent ici que les poissons, et supposent une morphologie de cours d'eau peu altérée ce qui n'est pas le cas général dans le bassin Seine-Normandie. Par ailleurs, un certain nombre de choix comme celui de représenter les étiages par le Q95 ou celui de s'intéresser à la perte d'habitat maximale des groupes considérés pourraient être discutés. De plus, les modèles de distribution des poissons et les seuils statistiques retenus pour prendre en compte les espèces font l'objet d'amélioration constante [Logez et al., 2012] et pourraient être affinés sur le bassin de la Seine. Enfin et surtout, s'il est important de quantifier l'altération d'habitat hydraulique, il faut garder en tête qu'elle ne constitue qu'un élément, non nécessairement limitant, des multiples altérations d'habitat subies par les organismes aquatiques [Leclere et al., 2012].

Malgré les limites de l'exercice, notre couplage de modèle fournit une vision globale de l'altération de l'habitat hydraulique, effet majeur induit par les prélèvements, et montre l'effet important du contexte biologique et du type de tronçon sur la traduction de l'altération hydrologique en altération d'habitat. Ainsi, l'application de modèles d'habitat à large échelle apporte une vision éco-hydrologique différente de la seule altération de débit. Ce type de couplage [Snelder et al., 2011a] ouvre la voie à la recherche de compromis " gagnants », par rapport à la situation actuelle, entre usages (prélèvements et leur réglementation) et milieux (habitat hydraulique des espèces).

\section{NOMENCLATURE}

$\triangle Q 95$ : Altération hydrologique du Q95 entre la situation naturalisée et influencée

$\triangle S P U$ : Altération habitat entre la situation naturalisée et influencée

$H$ : hauteur d'eau moyenne dans le tronçon de cours d'eau considéré (en $\mathrm{m}$ )

$L$ : largeur mouillée moyenne du tronçon de cours d'eau considéré (en m)

MECE : Masse d'Eau Cours d'Eau

MESO : Masse d'Eau Souterraine

Q95: Quantile de débit dépassé $95 \%$ du temps dans le cours d'eau considéré (en $\mathrm{m}^{3} / \mathrm{s}$ )

Q95i: Q95 influencé calculé à partir du débit $Q 95 n$ sur lequel est appliqué $\triangle Q 95\left(\mathrm{en} \mathrm{m}^{3} / \mathrm{s}\right)$

Q95n: Q95 naturalisé (sans influence anthropique) issu des simulations de Sauquet et Catalogne [2011] $\left(\mathrm{en} \mathrm{m}^{3} / \mathrm{s}\right)$

RHT : Réseau Hydrographique Théorique

RHT_DCE : Correspondance entre le réseau MECE et le réseau RHT

RHT_MECENa : Correspondance entre le réseau RHT et le réseau MECE où ont été réalisées des simulations hydrologiques [Flipo et al. 2013]

SPU : Surface Pondérée Utile (ou Surface Potentiellement Utilisable) en $\mathrm{m}^{2}$.

SPUi : SPU calculée pour le $Q 95 i$ (situation influencée) en $\mathrm{m}^{2}$ $S P U n$ : SPU calculée pour le $Q 95 n$ (situation naturalisée) en $\mathrm{m}^{2}$ $V$ : vitesse moyenne du tronçon de cours d'eau considéré (en $\mathrm{m} / \mathrm{s})$

$V H$ : Valeur d'Habitat (varie entre 0 et 1 )

\section{ANNEXE 1}

Équations de géométrie hydraulique (cf. [Lamouroux et al., 2010] pour les détails).

Les équations de géométrie hydraulique permettent d'estimer $L$ et $H$ par des lois puissance :

$$
L=a_{d} \bar{Q}^{b_{d}}\left[\frac{Q}{\bar{Q}}\right]^{b}
$$

où $\bar{Q}$ est le module interannuel. Le coefficient ad et les exposants de géométrie hydraulique longitudinale (bd) et stationnelle (b) sont soit des constantes, soit fonction de la pente des tronçons.

$$
H=c_{d} \bar{Q}^{f_{d}}\left[\frac{Q}{\bar{Q}}\right]^{f}
$$

Le coefficient cd et les exposants de géométrie hydraulique longitudinale (fd) et stationnelle (f) sont soit des constantes, soit fonction de la pente des tronçons.

\section{REFERENCES}

ACreman M.C., Ferguson A.J.D. (2010) - Environmental flows and the European Water Framework Directive. Freshwater Biology. Freshwater Biology. 55 32-48

Acreman M.C., Overton I.C., King J., Wood P., Cowx I.G., Dunbar M.J., Kendy E., Young W. (2014) — The changing role of ecohydrological science in guiding environmental flows. Hydrological Sciences Journal, 59 : 433450

Fielding A.H., Bell J.F. (1997) - A review of methods for the assessment of prediction errors in conservation presence/ absence models. Environmental Conservation. 24 : 3849

Flipo N., Monteil C., Poulin M., De Fouquet C., Krimissa M. (2012) - Hybrid fitting of a hydrosystem model: long term insight into the Beauce aquifer functioning (France). Water Resources Research. 48 : W05509

Flipo N., Labarthe B., Saleh F., Pryet A., Goblet P., Viennot P., ABAsQ L. (2013) - Relations eaux souterraines - réseau hydrographique sur le bassin Seine-Normandie : quantification des flux hydriques. Rapport final

GinOT V. (1998) - Logiciel EVHA. Evaluation de l'habitat physique des poissons en rivière (version2.0). Cemagref Lyon et Ministère de l'aménagement du Territoire et de l'Environnement, Direction de l'Eau, Paris, France

LAmouroux N., CAPRA H. (2002) - Simple predictions of instream habitat model outputs for target fish populations. Freshwater Biology. 47 1543-1556

LAMOUROUX N. (2008) - Hydraulic geometry of stream reaches and ecological implications. Gravel Bed Rivers 6: From Process Understanding to the Restoration of Mountain Rivers ; ed. : H. Habersack, H. Piégay, M. Rinaldi. Developments in Earth Surface Processes. 11 661-675

Lamouroux N. (2008) - ESTIMHAB. Free Excel sheet for cost-efficient instream flow studies (fish populations and guilds). Updated version. Cemagref-Conseil supérieur de la pêche

Lamouroux N., Pella H., Vanderbecq A., Sauquet E., Lejot J. (2010) - ESTIMKART 2.0 : Une plate-forme de modèles écohydrologiques pour contribuer à la gestion des cours d'eau à l'échelle des bassins français. Cemagref-Agence de l'Eau Rhône-Méditerranée-Corse - Onema. 50p 
Lamouroux N., Merigoux S., Doledec S., Snelder T.H. (2013) - Transferability of hydraulic preference models of aquatic macroinvertebrates. River Research and Applications. 29 933-937

Lamouroux N., Pella H., Snelder T.H., Sauquet E., Lejot J., SHANKAR U. (2014) - Uncertainty models for estimates of physical characteristics of river segments estimated over large areas. Journal of the American Water Resources Association. 50 1-13

LeClere J., Belliard J., OBerdorfF T. (2012) - Young-of-the-year fish assemblages as indicators of anthropogenic disturbances in large tributaries of the Seine River Basin (France). Hydrobiologia. 694 99-116

Leopold L.B., Maddock T. (1953) - The hydraulic geometry of stream channels and some physiographic implications

U.S. Geological Survey Professional Paper. 252

Logez M., Bady P., PonT D. (2012) - Modelling the habitat requirement of riverine fish species at the European scale: sensitivity to temperature and precipitation and associated uncertainty. Ecology of Freshwater Fish. 21 266-282

Oberdorff T., Pont D., Hugueny B., Chessel D. (2001) - A probabilistic model characterizing fish assemblages of French rivers: a framework for environmental assessment. Freshwater Biology. 46 399-415

PARK C.C. (1977) - World-wide variations in hydraulic geometry exponents of stream channels: an analysis and some observations. Journal of Hydrology. 33 133-146

Pella H., Lejot J., Lamouroux N., Snelder T. (2012) - Le réseau hydrographique théorique (RHT) français et ses attributs environnementaux. Géomorphologie : relief, processus, environnement. 3 317-336

Poff N.L., Richter B., Arthington A.H., Bunn S.E., Naiman R.J., Kendy E., Acreman M., Apse C., Bledsoe B.P., Freeman M., Henriksen J., Jacobson R.B., Kennen J., Merritt D.M., O'KeefFe J., Olden J.D., Rogers K., Tharme R.E., Warner A. (2010) — The ecological limits of hydrologic alteration
(ELOHA): a new framework for developing regional environmental flow standards. Freshwater Biology. 55 147-170

Pryet A., Labarthe B., Saleh F., Akopian M., Flipo N. (2015) Reporting of stream-aquifer flow distribution at the regional scale with a distributed process-based model. Water Resources Management. 29 139-159

Richter B.D., Baumgartner J.V., Powell J., And Braun D.P. (1996) - A method for assessing hydrologic alteration within ecosystems. Conservation Biology. 10 1163-1174

Saleh F., Flipo N., Habets F., Ducharne A., Oudin L., Viennot P., LEDOUX E. (2011) - Impact of in-stream water level fluctuations on interactions between streams and aquifer units at the regional scale. Journal of Hydrology. $\mathbf{4 0 0}$ 490-500

SAUQueT E. (2006) - Mapping mean annual river discharges: geostatistical developments for incorporating river network dependencies. Journal of Hydrology. 331 300-314

Sauquet E., Catalogne C. (2011) - Comparison of catchment grouping methods for flow duration curve estimation at ungauged sites in France. Hydrology and Earth System Sciences. 15 2421-2435

Snelder T.H., Booker D., Lamouroux N. (2011) - A method to assess and define environmental flow rules for large jurisdictional regions. Journal of the American Water Resources Association. 47 828-840

Snelder T.H., Lamouroux N., Pella H. (2011) - Empirical modelling of large scale patterns in river bed surface grain size

Geomorphology. 127 189-197

THARME R.E. (2003) - A global perspective on environmental flow assessment: emerging trends in the development and application environmental flow methodologies for rivers. River Research and Applications. 19 397-441

Wilding T.K., Bledsoe B., Poff N.L., SAnderson J. (2014) Predicting habitat response to flow using generalized habitat models for trout in Rocky Mountain streams. River Research and Applications. 7 805-824 